\title{
The use of vancomycin paste to reduce sternal wound infections after cardiac surgery-why is this still a "sticky" subject
}

\author{
Harold L. Lazar, MD
}

From the Division of Cardiac Surgery, Boston University School of Medicine, Boston, Mass.

Disclosures: Author has nothing to disclose with regard to commercial support.

Received for publication June 12, 2017; accepted for publication June 14, 2017; available ahead of print July 11, 2017

Address for reprints: Harold L. Lazar, MD, 88 East Newton St, Boston, MA 01228 (E-mail: Harold.1.lazar@ gmail.com).

J Thorac Cardiovasc Surg 2017;154:1324-5

0022-5223/\$36.00

Copyright (C) 2017 by The American Association for Thoracic Surgery

http://dx.doi.org/10.1016/j.jtcvs.2017.06.034

Preferred Items for Reporting Systemic Reviews and Meta-Analysis were developed to ensure that literature reviews are fully transparent and allow the readers to assess the strength and weakness of the literature being reviewed. ${ }^{1}$ As part of this process, it is important that the authors identify those articles that are a source of substantial heterogeneity that may affect the outcomes of the meta-analysis.

In this edition of the Journal, Kowalewski and colleagues $^{2}$ report the results of their meta-analysis to assess the effectiveness of vancomycin paste in reducing sternal wound infections (SWIs) after cardiac surgery. This study was undertaken after a recent study by Lander and colleagues $^{3}$ from the Brigham and Women's Hospital demonstrated that vancomycin paste did not reduce the incidence of deep sternal wound infections (DSWI) after cardiac surgery. This finding was contrary to other published studies on the use of vancomycin paste, which showed a significant reduction of all SWIs in patients undergoing cardiac surgery. The study by Lander and colleagues was significant in that it reported the largest series $(14,492$ patients) on this subject and, therefore, would have a major impact on the outcome of the meta-analysis.

The authors sought to analyze this study in more detail to determine why its conclusions differed from the other series, which all reported beneficial effects of vancomycin paste in reducing sternal infections. They found that the study had several methodologic flaws: (1) the study took place during a 12-year period (2003-2015) during which time there were numerous changes involving perioperative antibiotics, glycemic control, and sternal closure techniques. (2) Not all the surgeons used vancomycin paste, in fact; only $34.5 \%$ of the study patients received vancomycin paste. (3) The incidence of superficial SWIs, which also contribute to prolonged hospitalization, readmissions, and increased medical costs, were not reported. (4) Patients with infective endocarditis and those undergoing cardiac transplants were excluded from the analysis. These

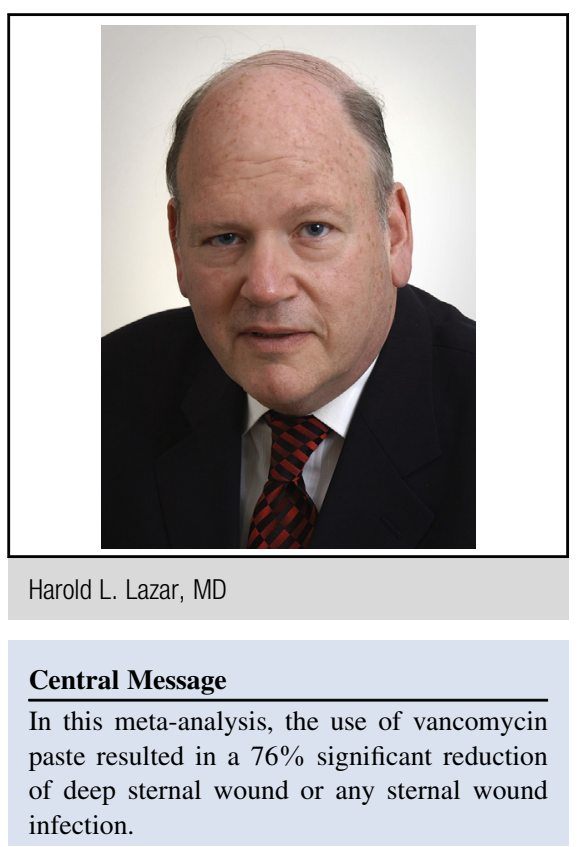

See Article page 1320 .

immunocompromised patients, many of whom also received steroids, stood to gain the most from vancomycin paste and were included in the other vancomycin studies. (5) Nine patients developed DSWI between 90 and 365 days from the time of surgery but were not included in the analysis. (6) Forty percent of the DSWI occurred in patients with diabetes, but no subgroup analyses were performed to determine whether vancomycin paste was protective in this high-risk group. Kowalewski and colleagues ${ }^{2}$ astutely pointed out that vancomycin paste was used in only $25 \%$ of patients from 2003 to 2010 when the DSWI rate was $1.29 \%$, but increased to $53 \%$ of patients from 2011 to 2015 when the DSWI rate fell to $0.29 \%$; a 5-fold decrease!

Despite these methodologic flaws, the study of Lander and colleagues met the criteria established by Kowalewski and colleagues ${ }^{2}$ for inclusion in their meta-analysis. Even with the inclusion of this flawed study, this meta-analysis has showed that the use of vancomycin paste was associated with a $76 \%$ significant risk reduction of any sternal wound infection and a $76 \%$ significant reduction in the incidence of DSWI. These results support the expert consensus review on prevention and management of SWIs, which assigned a 
Class I recommendation; Level of Evidence $=\mathrm{B}$; that "topical antibiotics be applied to the cut edges of the sternum on opening and before closing all cardiac surgical procedures involving a sternotomy." 4

Kowalewski and colleagues ${ }^{2}$ have shown us how a single flawed study may alter the outcomes of any meta-analysis. In a recent meta-analysis, Deppe and colleagues ${ }^{5}$ showed that the use of preoperative intra-aortic balloon pumps in high-risk patients, before coronary artery bypass surgery, decreased the risk of morbidity and mortality. The authors noted that this conclusion, which has a significant effect on clinical practice, previously had been questioned because of the inclusion of one study with significant methodologic flaws in a previous meta-analysis. How then can we avoid the inclusion of flawed methodologic studies that may meet inclusion criteria but can alter the conclusions of a meta-analysis?

The answer is not to publish them in the first place! When a manuscript is submitted for editorial review that has conclusions that differ from the existing literature, it should not necessarily be rejected; however, an attempt should be made to determine why these results were at odds with previous favorable studies on this subject. If the methodology and statistical analyses are sound, then the manuscript may offer an important alternative view and it should be accepted. However, if they are not, then publishing these manuscripts only serve to add unnecessary controversy to important areas that will influence clinical practice. Many of these "flawed" manuscripts are accompanied by an extensive limitations section, or an invited commentary pointing out the limitations of the study. However, after reading these reviews, one wonders why this manuscript was accepted in the first place! Perhaps, another criterion for acceptance of manuscripts by editorial boards should be "Is this the kind of manuscript that I would include if I was writing a meta-analysis on this subject?"

Finally, in view of the existing literature, why is the use of vancomycin paste still a "sticky" subject? Concerns have been raised, mostly by clinical pharmacologists, that vancomycin paste may increase serum levels of vancomycin for extended periods after surgery, which could predispose to the emergence of "resistant" bacterial organisms and renal and otic toxicity. To address these concerns, my colleagues and I performed a subanalysis of patients receiving vancomycin paste to measure serum levels of vancomycin in the postoperative period. ${ }^{6}$ We found that serum levels of vancomycin returned to baseline by the sixth postoperative day and that there was no increased incidence of postoperative resistant bacterial infections, renal, or otic toxicity for at least 90 days after surgery.

Others have criticized the paucity of randomized controlled trials (RCTs) to assess the efficacy of vancomycin paste. In fact, Kowalewski and colleagues ${ }^{2}$ mention an ongoing trial on the effect of topical vancomycin in reducing SWIs. Based on the results of the existing data, do we really need an RCT to prove that vancomycin paste is efficacious in reducing SWIs? In such a RCT, what would the control group be-the use of bone wax, an agent that prevents bone union, impairs osteogenesis and the ability to clear bacteria, has been shown to be an independent risk factor for sternal dehiscience and wound infections, ${ }^{7}$ and has received a Class III recommendation from the recent consensus guidelines on SWIs that it is "harmful and should not be applied on sternal edges." ${ }^{4}$ Vancomycin paste is relatively inexpensive, is easy to prepare and handle, and has no local or systemic side effects. It should be used in all patients undergoing a sternotomy if we are going to meet the goal of eliminating all SWIs after cardiac surgery.

\section{References}

1. Liberati A, Altman DG, Tetzlaff J, Mulrow C, Ioannidis JPA, Clarke M, et al The PRISMA statement for reporting systemic reviews and meta-analyses of studies that evaluate healthcare interventions. Ann Intern Med. 2009;151: 65-94.

2. Kowalewski M, Raffa GM, Szwed KA, Anisimowicz L. Meta-analysis to assess the effectiveness of topically used Vancomycin in reducing sternal wound infections after cardiac surgery. J Thorac Cardiovasc Surg. 2017; 154:1320-3.e3.

3. Lander HL, Ejiofor JI, McGurk S, Tsuyoshi K, Shekar P, Body SC. Vancomycin paste does not reduce the incidence of deep sternal wound infection after cardiac operations. Ann Thorac Surg. 2017; 103:497-503.

4. Lazar HL, Vander Salm T, Engelman R, Orgill D, Gordon S. Prevention and management of sternal wound infection. J Thorac Cardiovasc Surg. 2016; 152:962-72.

5. Deppe AC, Weber C, Liakopoulos J, Zeriouch M, Slotlosch I, Schemer M, et al Preoperative intra-aortic balloon pump use in high risk patients prior to coronary artery bypass surgery decreases the risk for morbidity and mortality-a metaanalysis of 9212 patients. J Card Surg. 2017;32:177-85.

6. Lazar HL, Barlam T, Cabral H. The effect of topical vancomycin applied to sternotomy incisions on postoperative serum vancomycin levels. J Card Surg. 2011; 26:461-5.

7. Prziborowski J, Hartrumpf M, Stock VA, Kuehnel RV, Albes JM. Is bone wax safe and does it help? Ann Thorac Surg. 2008;85:1002-6. 